\title{
An Exact Limit for the Peierls Distortion Energy in an Antiferromagnetic Chain by Means of a Renormalization Procedure
}

\author{
H. P. van de Braak, ${ }^{1}$ W. J. Caspers, ${ }^{1}$ F. W. Wiegel, ${ }^{1}$ and M. W. M. Willemse ${ }^{1}$
}

Received October 3, 1977

A renormalization procedure gives a rigorous upper bound for the groundstate energy per spin for a Peierls-distorted antiferromagnetic chain with Heisenberg interaction.

KEY WORDS: Peierls distortion; renormalization group; antiferromagnetic chain.

\section{INTRODUCTION}

Peierls distortions in a linear antiferromagnetic chain have been analyzed by a number of authors. ${ }^{(1-7)}$ This paper is devoted to the calculation of a rigorous upper bound for the ground-state energy per spin for the distorted chain. This calculation is based on a renormalization procedure developed in a previous paper ${ }^{(8)}$ which is used here in first order.

We consider an antiferromagnetic chain of a large number $N$ of quantum mechanical spins $\frac{1}{2}$ with alternating interaction constants depending on a distortion parameter $\xi$. The Hamiltonian of the spin system may be written ${ }^{(7)}$

$$
H\left(\xi, \mathbf{S}_{i}\right)=-J_{0} \sum_{i}\left[1+(-)^{i} \xi\right] \mathbf{S}_{i} \cdot \mathbf{S}_{i+1}, \quad J_{0}<0
$$

The distortion results in a classical elastic energy of the lattice ${ }^{(7)}$

$$
E_{l}(\Delta)=N \omega_{0} \Delta^{2}, \quad \xi=\gamma \Delta
$$

the kinetic energy of the lattice not being taken into account. In (2) the parameter $\Delta$ is the displacement of the lattice points, the relative change of the exchange constant being a linear function of this parameter.

\footnotetext{
${ }^{1}$ Department of Applied Physics, Twente University of Technology, Enschede, The Netherlands.
} 
In the next section we determine an upper bound for the lowest eigenvalue of (1) with our renormalization procedure and minimize the total energy per spin including (2), by varying $\xi$. In Section 3 a comparison with the results of other authors is made.

Because our results give an upper bound for the ground-state energy, we can prove rigorously that for $\omega_{0} / \gamma^{2} \leqslant 1.994$, Peierls distortion occurs.

\section{GROUND-STATE ENERGY PER SPIN}

As in Ref. 8, we divide the spin system into cells of three spins $(i=3 k-1,3 k, 3 k+1)$ and the energy eigenvalues for these cells are

$$
\begin{array}{ll}
-\frac{1}{2} J_{0} & \text { (quartet, } \left.S=\frac{3}{2}\right) \\
\frac{1}{2} J_{0}\left[1 \pm\left(1+3 \xi^{2}\right)^{1 / 2}\right] & \left(2 \text { doublets, } S=\frac{1}{2}\right)
\end{array}
$$

The lowest doublet for the $k$ th cell for all $\xi$ corresponds to an eigenvalue $\mathscr{E}=\frac{1}{2} J_{0}\left[1+\left(1+3 \xi^{2}\right)^{1 / 2}\right]$ and eigenstates

$$
\begin{aligned}
\left|\frac{1}{2}, \pm \frac{1}{2}\right\rangle_{k}= & (1 / \sqrt{6})\left[\mp 2 \alpha_{k}| \pm \mp \pm\rangle_{k} \pm\left(\alpha_{k}+\sqrt{3} \beta_{k}\right)|\mp \pm \pm\rangle_{k}\right. \\
& \left. \pm\left(\alpha_{k}-\sqrt{3} \beta_{k}\right)| \pm \pm \mp\rangle_{k}\right] \\
\alpha_{k}= & \frac{1}{\sqrt{2}} \frac{\eta_{k}}{\left[1+\eta_{k}{ }^{2}-\left(1+\eta_{k}{ }^{2}\right)^{1 / 2}\right]^{1 / 2}} \\
\beta_{k}= & \frac{1}{\sqrt{2}} \frac{1-\left(1+\eta_{k}{ }^{2}\right)^{1 / 2}}{\left[1+\eta_{k}{ }^{2}-\left(1+\eta_{k}\right)^{1 / 2}\right]^{1 / 2}} \\
\eta_{k}= & (-)^{k} \xi \sqrt{3}
\end{aligned}
$$

The symbols $|+-+\rangle_{k}$, etc., were introduced in Ref. 8 .

To determine the renormalized Hamiltonian for the spin system, we need the projected spin operators for the ground state

$$
\begin{aligned}
P_{1 / 2}(k) \mathbf{S}_{3 k k} P_{1 / 2}(k) & =\frac{1}{3}\left(-\alpha_{k}{ }^{2}+3 \beta_{k}{ }^{2}\right)\left(\mathbf{S}_{3 k-1}+\mathbf{S}_{3 k}+\mathbf{S}_{3 k+1}\right) P_{1 / 2}(k) \\
P_{1 / 2}(k) \mathbf{S}_{3 k \pm 1} P_{1 / 2}(k) & =\frac{2}{3} \alpha_{k}\left(\alpha_{k} \pm \sqrt{3} \beta_{k}\right)\left(\mathbf{S}_{3 k-1}+\mathbf{S}_{3 k}+\mathbf{S}_{3 k+1}\right) P_{1 / 2}(k)
\end{aligned}
$$

First-order perturbation calculus results in an effective Hamiltonian for the ground state in terms of the $\mathbf{S}_{k}^{(1)}=\left(\mathbf{S}_{3 k-1}+\mathbf{S}_{3 k}+\mathbf{S}_{3 k+1}\right) P_{1 / 2}(k)$ :

with

$$
H^{(1)}\left(\xi^{(1)}, \mathbf{S}_{k}^{(1)}\right)=\epsilon_{0}(\xi) N+c_{0}(\xi) H\left(\xi^{(1)}, \mathbf{S}_{k}^{(1)}\right)
$$

$$
\begin{aligned}
\epsilon_{0}(\xi) & =\frac{1}{6} J_{0}\left[1+\left(1+3 \xi^{2}\right)^{1 / 2}\right] \\
c_{0}(\xi) & =\frac{2 \xi^{2}}{\left[1+3 \xi^{2}-\left(1+3 \xi^{2}\right)^{1 / 2}\right]^{2}}\left[1+\xi^{2}-\left(1-\xi^{2}\right)\left(1+3 \xi^{2}\right)^{1 / 2}\right] \\
\xi^{(1)} & =\Gamma(\xi)=-\frac{2 \xi^{3}}{1+\xi^{2}-\left(1-\xi^{2}\right)\left(1+3 \xi^{2}\right)^{1 / 2}}
\end{aligned}
$$


The renormalization procedure leading to $H^{(1)}$ may be repeated, yielding a series expansion for the ground-state energy per spin

$$
\epsilon(\xi)=\sum_{s=0}^{\infty} \frac{1}{3^{s}}\left[\prod_{t=0}^{s-1} c_{0}\left(\xi^{(t)}\right)\right] \epsilon_{0}\left(\xi^{(s)}\right), \quad \xi^{(s)}=\Gamma\left(\xi^{(s-1)}\right), \quad \xi^{(0)}=\xi
$$

As was discussed in Ref. 8, first-order perturbation calculus leading to $\epsilon(\xi)$ gives a rigorous upper bound for the energy per spin.

The actual Peierls distortion is determined by the minimum of the total energy per spin, including the elastic energy (2), i.e., the minimum of

$$
w(\xi)=\epsilon(\xi)+\frac{E_{l}(\Delta)}{N}=\epsilon(\xi)+\frac{\omega_{0}}{\gamma^{2}} \xi^{2}
$$

Numerical results of our method are shown in four graphs and two tables. Figure 1 shows the graph of $\Gamma(\xi)$, which we need for the calculation of $\epsilon(\xi)$. We confine ourselves to the interval $[0,1.5]$ for $\xi, \Gamma(\xi)$ being an odd function. Asymptotic values of $\Gamma(\xi)$ are $\Gamma(\xi) \sim-4 \xi(\xi \rightarrow 0)$ and $\Gamma(\xi) \sim-2 / \sqrt{ } 3=$ $-1.1547(\xi \rightarrow \infty)$. One renormalization transformation gives a change of sign of the distortion parameter. Fixed points are given by the equation $\Gamma(\xi)=-\xi$ and the only ones for $\xi \geqslant 0$ are $\xi=0,1$. The stable point

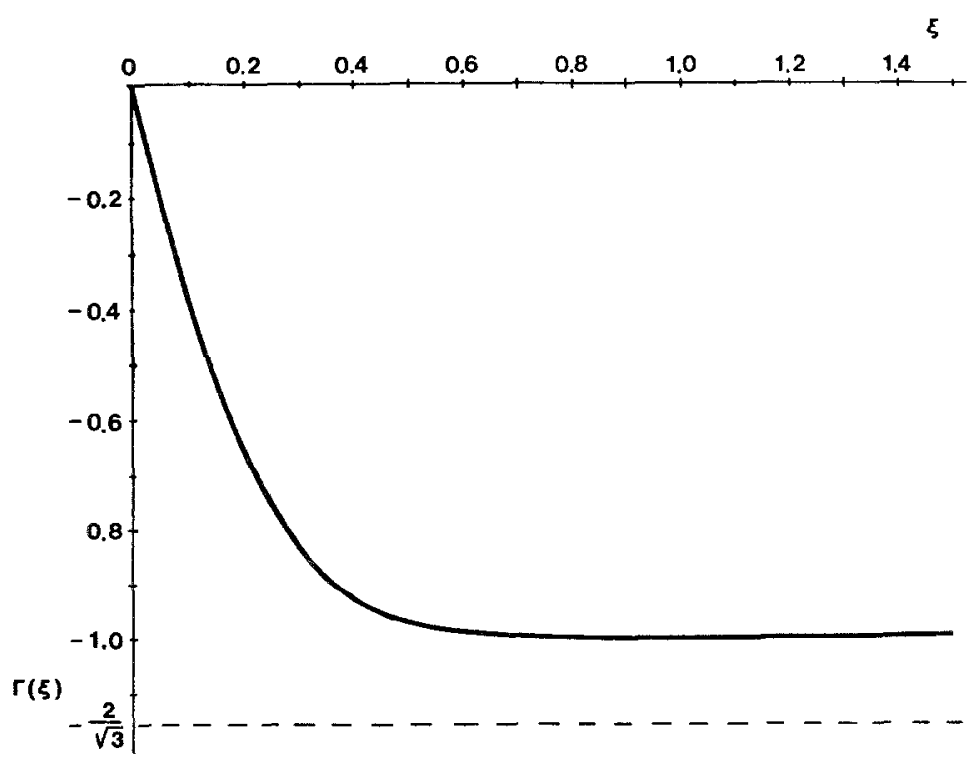

Fig. 1. The function $\Gamma(\xi)$. 


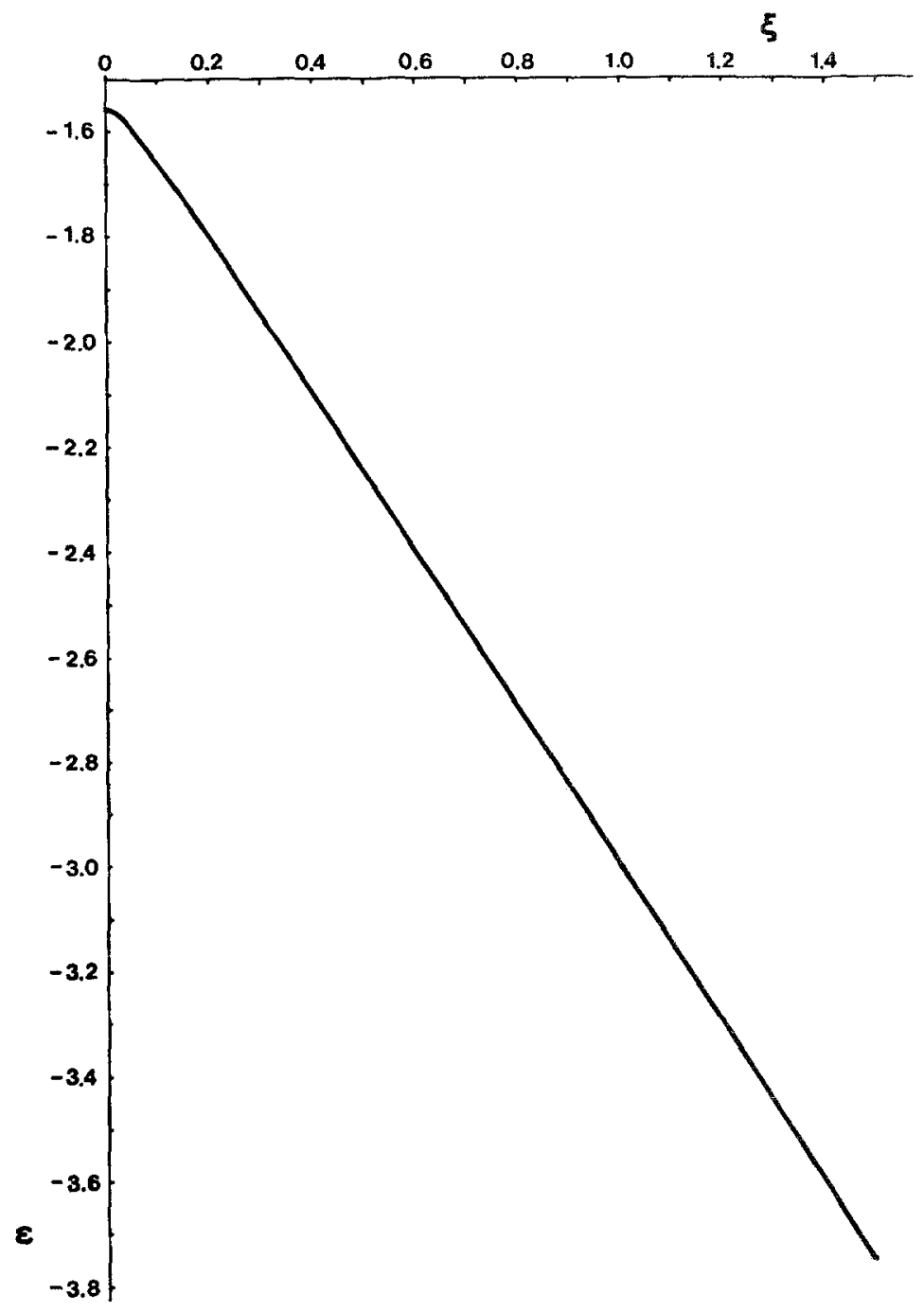

Fig. 2. The ground-state energy per spin $\epsilon(\xi)$.

$\left|\xi^{(s)}\right|=1$ is reached with sufficient accuracy after a small number of transformations, for all values of $\xi^{(0)}=\xi$ in the interval $[0,1.5]$. In Fig. 2 we give the values of $\epsilon(\xi)$ for $J_{0}=-4$.

For the fixed points we find $\epsilon(0)=-36 / 23=-1.5652$ and $\epsilon(1)=-3$. For the calculation of $\epsilon(\xi)$ for general values of $\xi$ use has been made of the Wang 500 desk calculator. The function $\epsilon(\xi)$ is monotonic for $\xi \geqslant 0$ and its behavior for $\xi \gtrsim 0$ is determined by a characteristic exponent $\alpha$, which is 
calculated as follows: We introduce three new functions $\bar{\epsilon}(\xi), \bar{c}_{0}(\xi)$, and $\bar{\epsilon}_{0}(\xi)$,

$$
\begin{aligned}
\epsilon(\xi)-\epsilon(0)=\epsilon(\xi)+36 / 23 & =\bar{\epsilon}(\xi) \\
c_{0}(\xi)-c_{0}(0)=c_{0}(\xi)-\frac{4}{9} & =\bar{c}_{0}(\xi) \\
\epsilon_{0}(\xi)-\epsilon_{0}(0)=\epsilon_{0}(\xi)+\frac{4}{3} & =\bar{\epsilon}_{0}(\xi)
\end{aligned}
$$

The function $\epsilon(\xi)$, according to (5), obeys the relation

$$
\epsilon(\xi)-\epsilon_{0}(\xi)=\frac{1}{3} c_{0}(\xi) \epsilon\left(\xi^{\prime}\right), \quad \xi^{\prime}=\Gamma(\xi)
$$

which may be translated in terms of the functions $\bar{\epsilon}(\xi), \bar{c}_{0}(\xi)$, and $\bar{\epsilon}_{0}(\xi)$

$$
\bar{\epsilon}(\xi)-\bar{\epsilon}_{0}(\xi)=-(12 / 23) \bar{c}_{0}(\xi)+(4 / 27) \bar{\epsilon}\left(\xi^{\prime}\right)+\frac{1}{3} \bar{c}_{0}(\xi) \bar{\epsilon}\left(\xi^{\prime}\right)
$$

For small $\xi$ the functions $\bar{\epsilon}_{0}(\xi)$ and $\bar{c}_{0}(\xi)$ are quadratic, whereas $\xi^{\prime} \sim-4 \xi$.

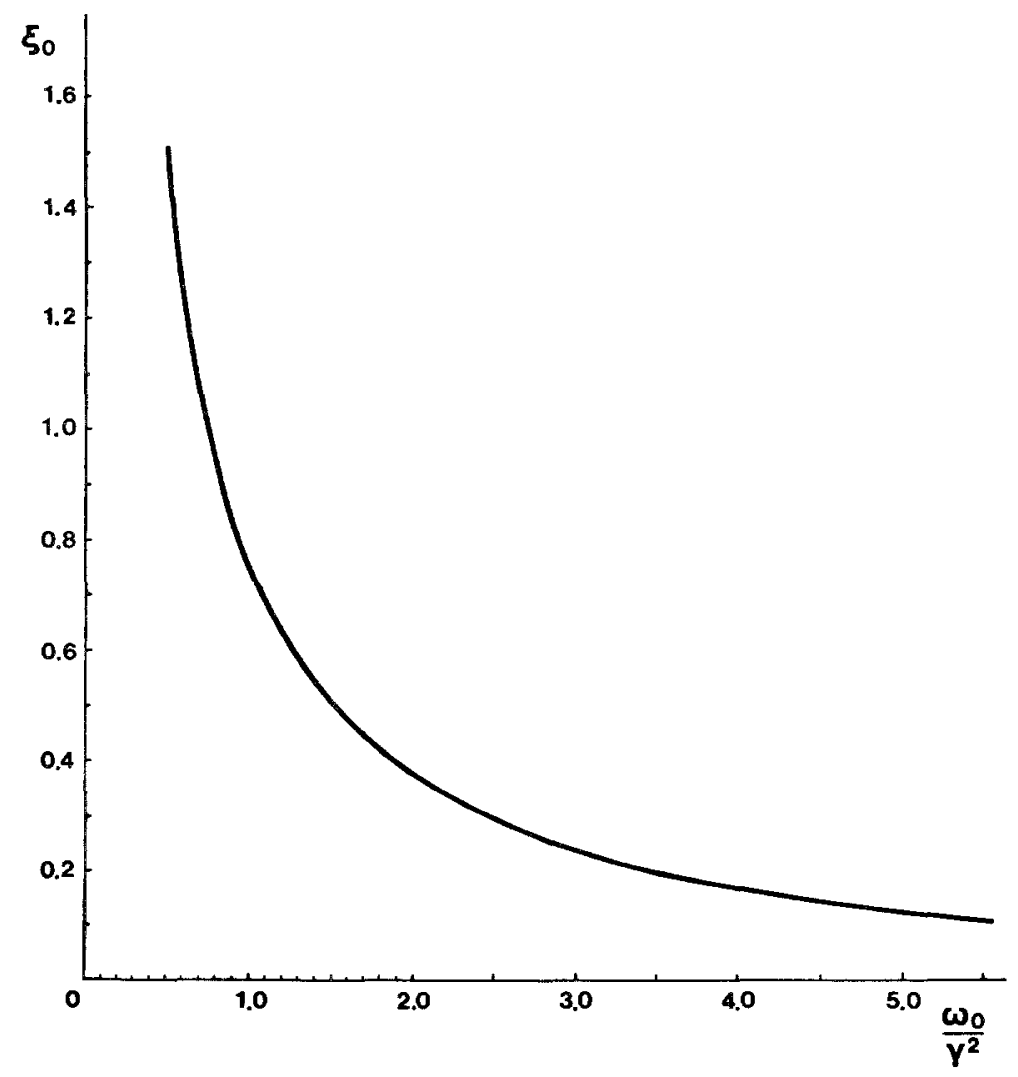

Fig. 3. Distortion parameter $\xi_{0}$ for minimum energy, as a function of $\omega_{0} / \gamma^{2}$. 
Introducing the exponent $\alpha$ by $\bar{\epsilon}(\xi) \sim k_{1}|\xi|^{\alpha}$, one easily determines the dominant terms in (7) for small $\xi$, which give the equation

$$
k_{1}|\xi|^{\alpha}=\frac{4}{27} k_{1} 4^{\alpha}|\xi|^{\alpha}, \quad \alpha=\frac{3 \ln 3}{2 \ln 2}-1=1.37744
$$

Because $\alpha<2$ the term $\bar{\epsilon}_{0}(\xi)$ of the left-hand side and the first and third terms of the right-hand side of (7) do not contribute to the dominant part. This value of $\alpha$ is confirmed by our numerical results for $\epsilon(\xi)$. For large values of $\xi, \xi^{\prime} \sim-2 / \sqrt{3}, c_{0}(\xi) \sim \frac{2}{9} \xi \sqrt{3}$, and

$$
\epsilon(\xi)=\epsilon_{0}(\xi)+\frac{1}{3} c_{0}(\xi) \epsilon\left(\xi^{\prime}\right) \sim\left[-\frac{2}{3} \sqrt{3}+\frac{1}{3} \frac{2}{9} \sqrt{3} \epsilon(-2 / \sqrt{3})\right] \xi=-1.56960 \xi
$$

The minimum of $w(\xi)$ [cf. formula (6)] is easily determined numerically and the value $\xi_{0}$ of $\xi$ for which this minimum is reached, as a function of $\omega_{0} / \gamma^{2}$, is given in Fig. 3. Finally Fig. 4 shows the graph of $w\left(\xi_{0}\right)$, the minimal

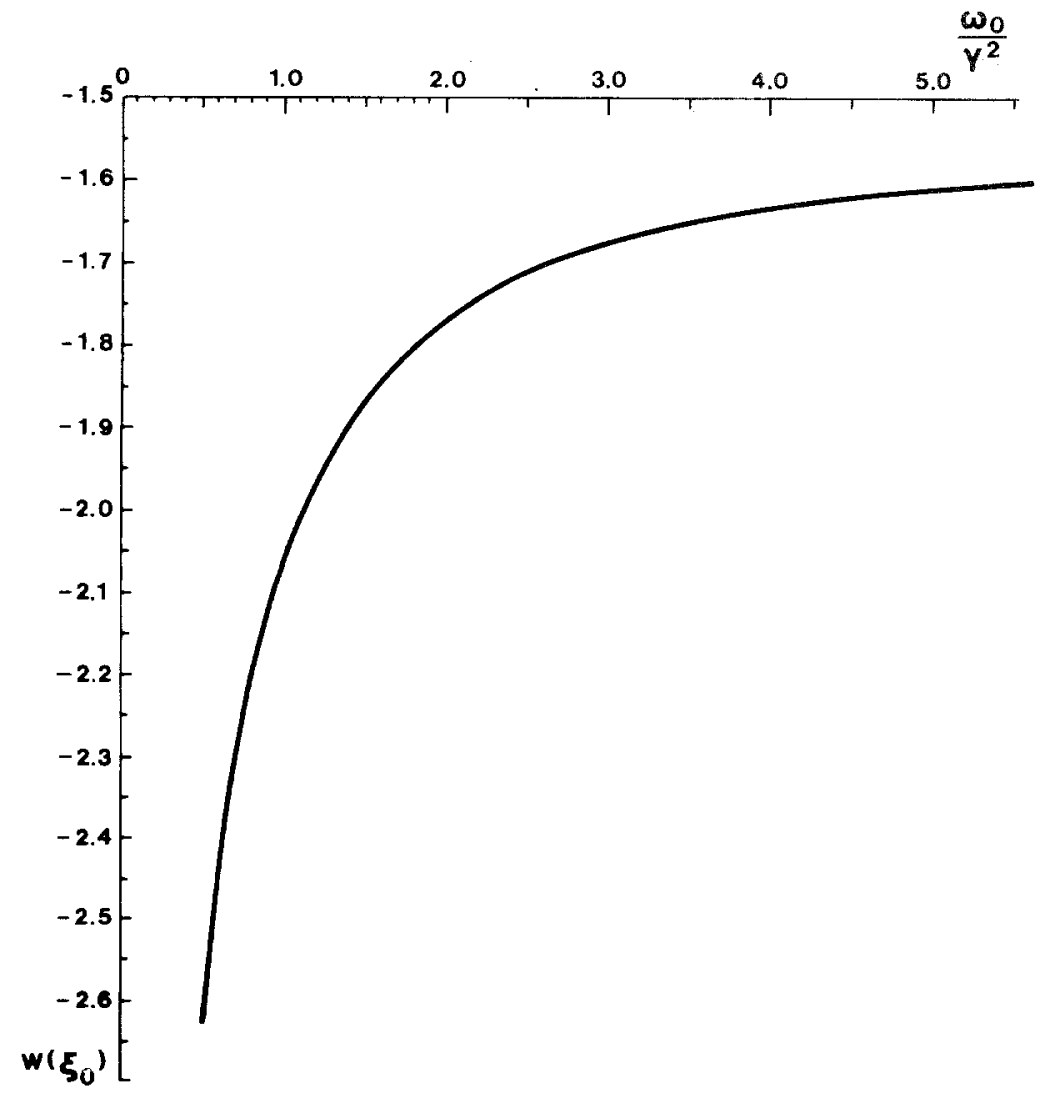

Fig. 4. Minimal energy $w\left(\xi_{0}\right)$ as a function of $\omega_{0} / \gamma^{2}$. 
Table I. $\xi_{0}$ As a Function of $\omega_{0} / \gamma^{2}$

\begin{tabular}{cccc}
\hline$\omega_{0} / \gamma^{2}$ & $\xi_{0}$ & $\omega_{0} / \gamma^{2}$ & $\xi_{0}$ \\
\hline 0.5 & 1.5125 & 1.5 & 0.5104 \\
0.6 & 1.2536 & 2.0 & 0.3826 \\
0.7 & 1.0714 & 2.5 & 0.3007 \\
0.8 & 0.9377 & 3.0 & 0.2436 \\
0.9 & 0.8351 & 3.5 & 0.2021 \\
1.0 & 0.7538 & 4.0 & 0.1713 \\
1.1 & 0.6877 & 4.5 & 0.1477 \\
1.2 & 0.6327 & 5.0 & 0.1293 \\
1.3 & 0.5859 & 5.5 & 0.1145 \\
1.4 & 0.5456 & & \\
\hline
\end{tabular}

energy of the distorted chain, as a function of $\omega_{0} / \gamma^{2}$. The results of Figs. 3 and 4 are also given in Tables $I$ and II. In addition to these results Table II gives values of $\epsilon\left(\xi_{0}\right)$.

\section{DISCUSSION}

We should stress that, in our approximation, there is a finite distortion for all finite values $\omega_{0} / \gamma^{2}$, as a consequence of the characteristic exponent $\alpha$ being smaller than 2 .

In our normalization, Hulthén's result ${ }^{(9)}$ equals $\epsilon_{H}=-1.7726$. Our calculations show $w\left(\xi_{0}\right)<\epsilon_{H}(0)$ for $\omega_{0} / \gamma^{2} \leqslant 1.994$, so that for these values of $\omega_{0} / \gamma^{2}$ one is sure that Peierls distortion occurs for the exact ground state, because our value for $w\left(\xi_{0}\right)$, being an upper bound for the energy, is smaller than Hulthén's result.

Table II. $w\left(\xi_{0}\right)$ and $\epsilon\left(\xi_{0}\right)$ as a Function of $\omega_{0} / \gamma^{2}$

\begin{tabular}{cccccc}
\hline$\omega_{0} / \gamma^{2}$ & $w\left(\xi_{0}\right)$ & $\epsilon\left(\xi_{0}\right)$ & $\omega_{0} / \gamma^{2}$ & $w\left(\xi_{0}\right)$ & $\epsilon\left(\xi_{0}\right)$ \\
\hline 0.5 & -2.6275 & -3.7713 & 1.5 & -1.8697 & -2.2604 \\
0.6 & -2.4379 & -3.3807 & 2.0 & -1.7717 & -2.0645 \\
0.7 & -2.3036 & -3.1071 & 2.5 & -1.7140 & -1.9400 \\
0.8 & -2.2031 & -2.9066 & 3.0 & -1.6772 & -1.8552 \\
0.9 & -2.1248 & -2.7525 & 3.5 & -1.6526 & -1.7956 \\
1.0 & -2.0619 & -2.6301 & 4.0 & -1.6352 & -1.7526 \\
1.1 & -2.0101 & -2.5303 & 4.5 & -1.6226 & -1.7208 \\
1.2 & -1.9666 & -2.4469 & 5.0 & -1.6130 & -1.6966 \\
1.3 & -1.9295 & -2.3758 & 5.5 & -1.6056 & -1.6777 \\
1.4 & -1.8975 & -2.3143 & & & \\
\hline
\end{tabular}


For $\omega_{0} / \gamma^{2}>1.994$ one cannot be sure of the occurrence of a distortion on the basis of our approximation, but we expect that for the exact solution of the problem one finds a distortion for all $\omega_{0} / \gamma^{2}$.

We may also compare our result for the rigid lattice $\epsilon(0)=-36 / 23=$ $-1.5652\left(\omega_{0}=\infty\right)$ with Hulthén's result. For this case, however, a far better approximation was given in a previous paper. ${ }^{(8)}$

Our results for $\xi_{0}$ given in Fig. 3 and Table I may be compared with those in Refs. 1 and 4 . In the first paper the analogous problem for the $X Y$ model is solved exactly, resulting in $\xi_{0} \approx \exp \left(-\pi \omega_{0} / 4 \gamma^{2}\right)\left(\xi_{0} \ll 1\right)$, expressed in terms of the corresponding constants in our analysis. For the Heisenberg interaction in the Hartree-Fock approximation it was found in Ref. 4 that $\xi_{0} \approx \exp (-\bar{a})\left(\xi_{0} \ll 1\right)$, where $\bar{a}=2 a /\left[1+(1+8 a / \pi)^{1 / 2}\right]$ and $a=\pi \omega_{0} / 4 \gamma^{2}$.

Pytte $^{(5)}$ has analyzed the Peierls distortion for a linear antiferromagnetic chain in interaction with three-dimensional phonons. Jacobs et al ${ }^{(6)}$ have given a survey of the energy lowering of the ground state near the uniform limit $(\xi=0)$ : For the $X Y$ model it goes like $\xi^{2} \ln \xi^{(1,3)}$ and for the Heisenberg model in the HF approximation like $\xi^{2}(\ln \xi)^{2}{ }^{(4,5)}$ whereas in our first-order approximation for small cells of three spins we find $\xi^{1.37744}$.

\section{ACKNOWLEDGMENT}

The authors wish to thank Prof. J. Kommandeur of the Chemistry Department of the University of Groningen, who suggested the problem.

\section{REFERENCES}

1. P. Pincus, Solid State Comm. 9:1971 (1971).

2. G. Beni and P. Pincus, J. Chem. Phys. 57:3531 (1972).

3. J. Y. Dubois and J. B. Carton, J. Phys. (Paris) 35:371 (1974).

4. G. Beni, J. Chem. Phys. 58:3200 (1973).

5. E. Pytte, Phys. Rev. B 10:4637 (1974).

6. I. S. Jacobs, J. W. Bray, H. R. Hart, Jr., L. V. Interrante, J. S. Kasper, G. D. Watkins, D. E. Prober, and J. C. Bonner, Phys. Rev. B 14:3036 (1976).

7. K. A. Penson, A. Holz, and K. H. Benneman, in Proc. International Conference on Magnetism ICM-76, III (Amsterdam, 1976), p. 1135.

8. H. P. van de Braak, W. J. Caspers, C. de Lange, and M. W. M. Willemse, Physica 87A : 354 (1977).

9. L. Hulthén, Ark. Math. Astron. Fysik 26A, no. 11 (1938); F. Keffer, Encyclopedia of Physics XVIII/2 (Springer, Berlin, 1966), 1. 